\title{
In Vivo Imaging of Glycol Chitosan-Based Nanogel Biodistribution
}

\author{
Paula Pereira, Alexandra Correia, Francisco M. Gama*
}

The preclinical development of nanomedicines raises several challenges and requires a comprehensive characterization. Among them is the evaluation of the biodistribution following systemic administration. In previous work, the biocompatibility and in vitro targeting ability of a glycol chitosan (GC) based nanogel have been validated. In the present study, its biodistribution in the mice is assessed, using near-infrared (NIR) fluorescence imaging as a tool to track the nanogel over time, after intravenous administration. Rapid whole body biodistribution of both Cy5.5 labeled GC nanogel and free polymer is found at early times. It remains widespreadly distributed in the body at least up to $6 \mathrm{~h}$ postinjection and its concentration then decreases drastically after $24 \mathrm{~h}$. Nanogel blood circulation half-life lies around $2 \mathrm{~h}$ with the free linear GC polymer presenting lower blood clearance rate. After $24 \mathrm{~h}$, the blood NIR fluorescence intensity associated with both samples decreases to insignificant values. NIR imaging of the organs shows that the nanogel had a body clearance time of $\approx 48 \mathrm{~h}$, because at this time point a weak signal of NIR fluorescence is observed only in the kidneys. Hereupon it can be concluded that the engineered GC nanogel has a fairly long blood circulation time, suitable for biomedical applications, namely, drug delivery, simultaneously allowing efficient and quick body clearance.

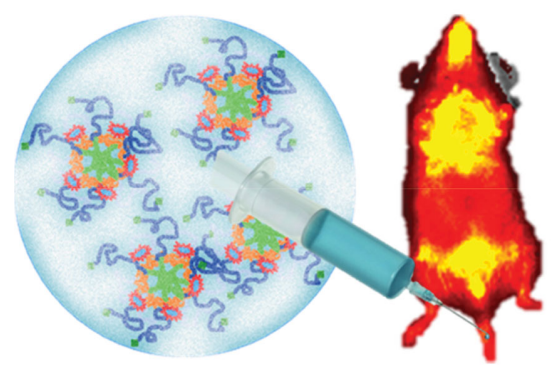

\section{Introduction}

The use of engineered nanoparticles (NPs) in nanomedicine is revolutionizing the clinical practice, regarding both diagnosis and therapy. ${ }^{[1]}$ Due to their multifunctional nature, large surface area, structural diversity, and long vascular circulation time (as compared to small molecules), NPs have

P. Pereira, F. M. Gama

Centre of Biological Engineering

University of Minho

Campus de Gualtar, 4710-057 Braga, Portugal

E-mail: fmgama@deb.uminho.pt

A. Correia

I3S - Instituto de Investigação e Inovação em Saúde

Universidade do Porto and IBMC - Instituto de Biologia

Molecular e Celular

Rua do Campo Alegre, 4099-003 Porto, Portugal emerged as attractive vehicles for improved therapy. In addition to being used as tools for molecular imaging they also could deliver therapeutic agents to the injury site, thus allowing simultaneously imaging and therapy, called theragnosis. ${ }^{[2,3]}$ Such nanoparticulate systems should enable appropriate residence time in blood stream, long enough as to afford delivery of the drug and/or diagnostic probes at target site, at the same time allowing its complete elimination within an acceptable timeframe, in order to avoid toxicity or chronic effects. ${ }^{[4,5]}$ Unfortunately, many types of systemically injected NPs have a rapid blood clearance, essentially due to the action of the mononuclear phagocytic system mainly at the liver, spleen, lung, and bone marrow. So, NPs formulation that avoids rapid clearance is a requirement for suitable delivery to the desired target. [5]

In a previous study we reported the synthesis of a glycol chitosan (GC) nanogel functionalized with folate with an average size of $200 \mathrm{~nm}$ and positive surface charge 
$(+25 \mathrm{mV}) \cdot{ }^{[6]}$ In vitro assays confirmed ability for folate receptors targeting and also the efficient encapsulation of siRNA. Few studies of chitosan based NPs biodistribution are available in literature, most of which are focused on anti-tumor efficacy of a drug loaded NP. ${ }^{[7]}$ Thus in the current work we intend to show an assessment of the GC nanogel biodistribution in a mice model, using optical fluorescence imaging technology. Near-infrared (NIR) based imaging, in addition to enabling noninvasive study of molecular targets inside the body of the living animal, is versatile, easy-of-use, avoids the use of radiopharmaceuticals, and has a relatively low cost. ${ }^{[8,9]}$ The NIR probe Cy5.5 was chosen to label the nanogel as well as the free polymer. Fluorophores with a red or NIR emission range (600-1000 nm) bear a high photon penetration into living tissues, and low photon absorption and tissue autofluorescence, thus allowing effective imaging of deep tissues. ${ }^{[0,11]}$

\section{Experimental Section}

Materials: GC (G7753, $\left.M_{\mathrm{w}}=100 \mathrm{kDa}\right)$, mercapto hexadecanoic acid (MHDA), $N$-hydroxysulfosuccinimide (NHS), 1-Ethyl3-[3-dimethylaminopropyl]carbodiimide hydrochloride, folate, O-methyl-O'-succinylpolyethyene glycol 2000 (PEG2000), and
O-(2-Aminoethyl)-O'-(2-carboxyethyl)polyethylene glycol 3000 hydrochloride (PEG3000) were acquired from Sigma-Aldrich (St. Louis, MO, USA). Cy5.5 monoreactive NHS ester was purchased from GE Healthcare (Little Chalfont, UK).

Synthesis and Self-Assembly of GC Nanogels: Details on the synthesis of the GC nanogel synthesis and its decoration with folic acid were described in a previous report. ${ }^{[6]}$ Briefly, nanogel synthesis was performed in two independent steps. Initially, folate was conjugated to PEG3000 (FA-PEG3000). In the second reaction, FA-PEG3000, PEG2000, and MHDA were grafted onto the GC polymer. The nanogel colloidal suspensions were obtained after dispersing the lyophilized reaction product in saline buffer (PBS), under magnetic stirring at $50{ }^{\circ} \mathrm{C}$ for $48 \mathrm{~h}$, and filtration through a pore size $0.45 \mu \mathrm{m}$ cellulose acetate syringe filter.

In Vitro Evaluation of Nanogel Serum Stability: The nanogel serum stability was assessed by incubating the nanogel at a concentration of $1 \mathrm{mg} \mathrm{mL}^{-1}$ in PBS with $1 \%$ (v/v) of fetal bovine serum (FBS), under constant stirring at room temperature. At predefined time points $(0,2$, and $24 \mathrm{~h}$ ) the samples size distribution was monitored by DLS using a Malvern Nano ZS instrument (Malvern Instruments).

Preparation of GC and Nanogel Cy5.5 Conjugation: Free polymer and nanogels were labeled with an NIR fluorescent probe, Cy5.5 monoreactive NHS ester. The chemical structure of Cy5.5 conjugated with nanogel is shown in Scheme 1. The dye solution (10 $\mathrm{mg} \mathrm{mL}^{-1}$, in DMSO) was added to GC or nanogel dispersion ( $1 \mathrm{mg} \mathrm{mL}^{-1}$, in PBS) at 0.04 and 0.06 molar ratios of

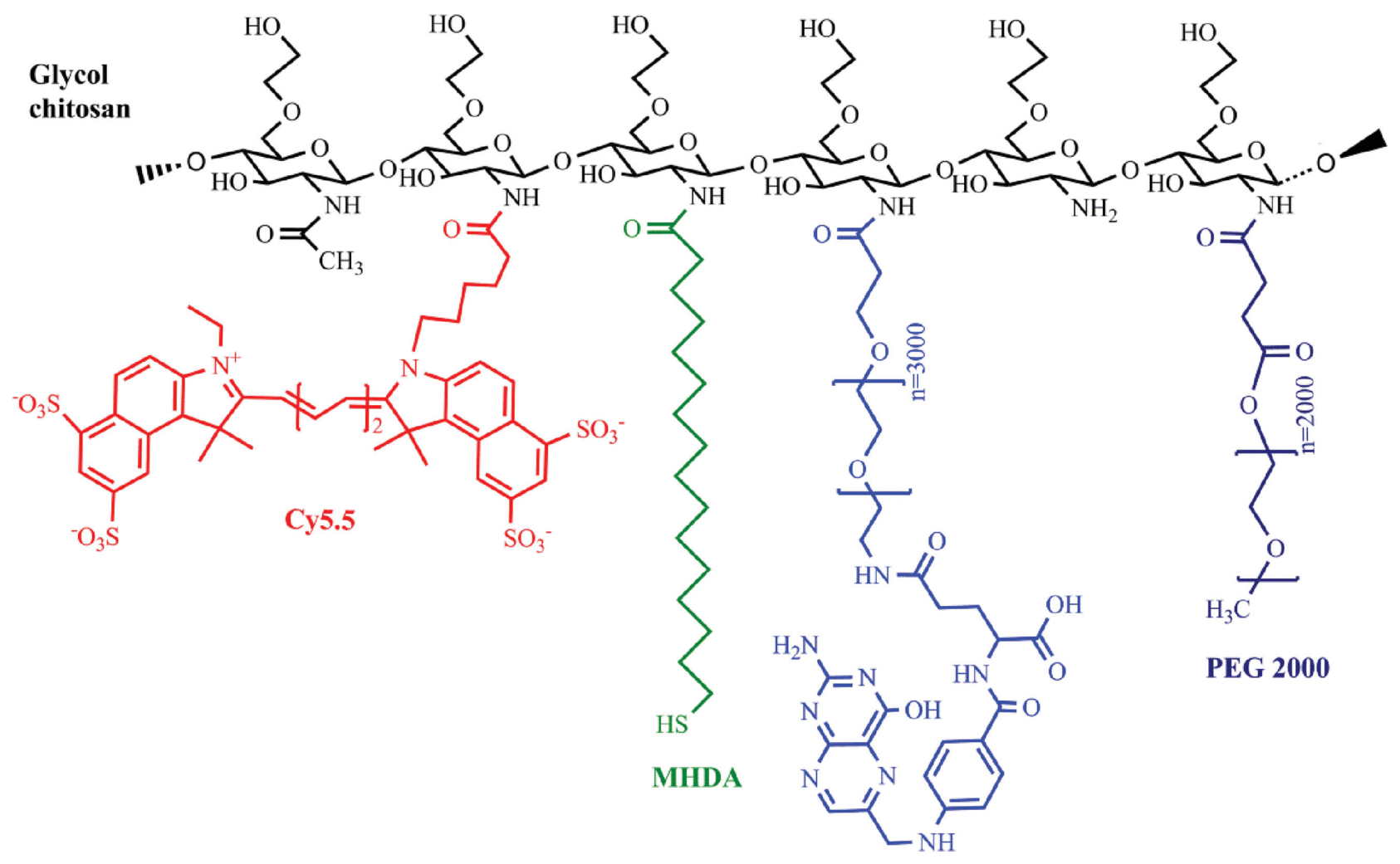

FA-PEG 3000

Scheme 1. Representation of the chemical structure of Cy5.5 conjugated with nanogel.

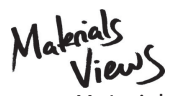

www.MaterialsViews.com 
Cy5.5 reactive carboxylic groups to GC or nanogel free amine groups, respectively. The reaction was allowed to occur for $24 \mathrm{~h}$ in the dark at room temperature. Thereafter, the reaction mixture was extensively dialyzed $\left(M_{\mathrm{w}}\right.$ cutoff $\left.10-12 \mathrm{kDa}\right)$ against distilled water to remove unreacted Cy5.5 molecules. To confirm the absence of free dye, Cy5.5-GC or Cy5.5-Nanogel was purified by centrifugation at $3000 \times \mathrm{g}$ through a $10 \mathrm{kDa} M_{\mathrm{w}}$ cutoff filter. Then, Cy5.5 was quantified spectrophotometrically in the resultant fractions (filtrate and concentrate).

In Vivo Biodistribution of GC and GC Nanogels: The animal experiments were performed in agreement and approved by IMM (Institute of medicine and Molecular, Lisbon, Portugal) Animal Ethics Committee and Portuguese General Alimentary and Veterinarian Board (authorization number 006315/27/03/2014, from DGAV-Portugal). Male BALB/c mice, eight weeks old (Charles River, L'Arbresle, France), were used for

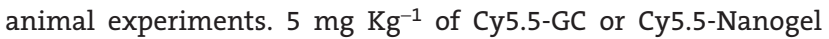
was intravenously injected via the tail vein. After injection, the time-dependent in vivo samples biodistribution were noninvasively imaged with mice anesthetized with Ketamine $75 \mathrm{mg} \mathrm{kg}^{-1}$ BW and Medetomidine $1 \mathrm{mg} \mathrm{Kg}^{-1}$ BW solution, using the IVIS Lumina fluorescence imaging system.

For the imaging study, exposure time (3 s), pixel binning (CCD resolution, medium), and lens aperture (amount of light collected and depth-of-field, f/stop 16) were optimized. The NIR fluorescence signal intensity of each of the Cy5.5 labeled samples on respective injected animals was imaged using a CCD camera equipped with a Cy5.5 bandpass emission filter (680-720 nm). The obtained results were resultant from two independent experiments, with consistent results being obtained. Since only a qualitative analysis of the results was performed, it was decided not to perform additional replicates.

Fluorescence Intensity Measurement in the Blood and Different Organs: At the end of each time point whole blood (about $0.8 \mathrm{~mL}$ ) was collected from anesthetized animals using cardiac puncture. Immediately after, they were sacrificed through cervical dislocation to collect the organs of interest: the spleen, heart, liver, kidneys, brain, lungs, muscle, and skin. Blood and organs were placed in a 6-well plate for IVIS Lumina fluorescence imaging system visualization. The semi-quantification of NIR fluorescence signal in different organs and blood acquired images was performed using a Living image software and measured as total photons per centimeter squared per steradian $\left(\mathrm{p} \mathrm{s}^{-1} \mathrm{~cm}^{-2} \mathrm{sr}^{-1}\right)$.

\section{Results and Discussion}

\subsection{Nanogel Serum Stability}

It is known that positively charged particles (such as GC nanogel, which has a potential zeta of $+25 \mathrm{mV}^{[6]}$ easily induce nonspecific interactions with serum proteins, which can contribute to nanogel colloidal disassembling, rapid blood clearance, and also to opsonization and liver accumulation. ${ }^{[12,13]}$ The assessment of GC nanogel interaction with FBS was studied by dynamic light scattering. As can be observed in Figure 1, the nanogel showed colloidal stability and constant size in the presence of FBS, even after $24 \mathrm{~h}$ of incubation. Indeed, the nanogel average size, around $200 \mathrm{~nm}$, remained consistent over the time. It can also be seen that serum proteins are somewhat unstable, likely due to aggregation, as noted by the shift toward a higher size distribution. This result emphasizes the absence of extensive interaction between nanogel and serum components, probably due to its PEG functionalization, since it is well-known the ability
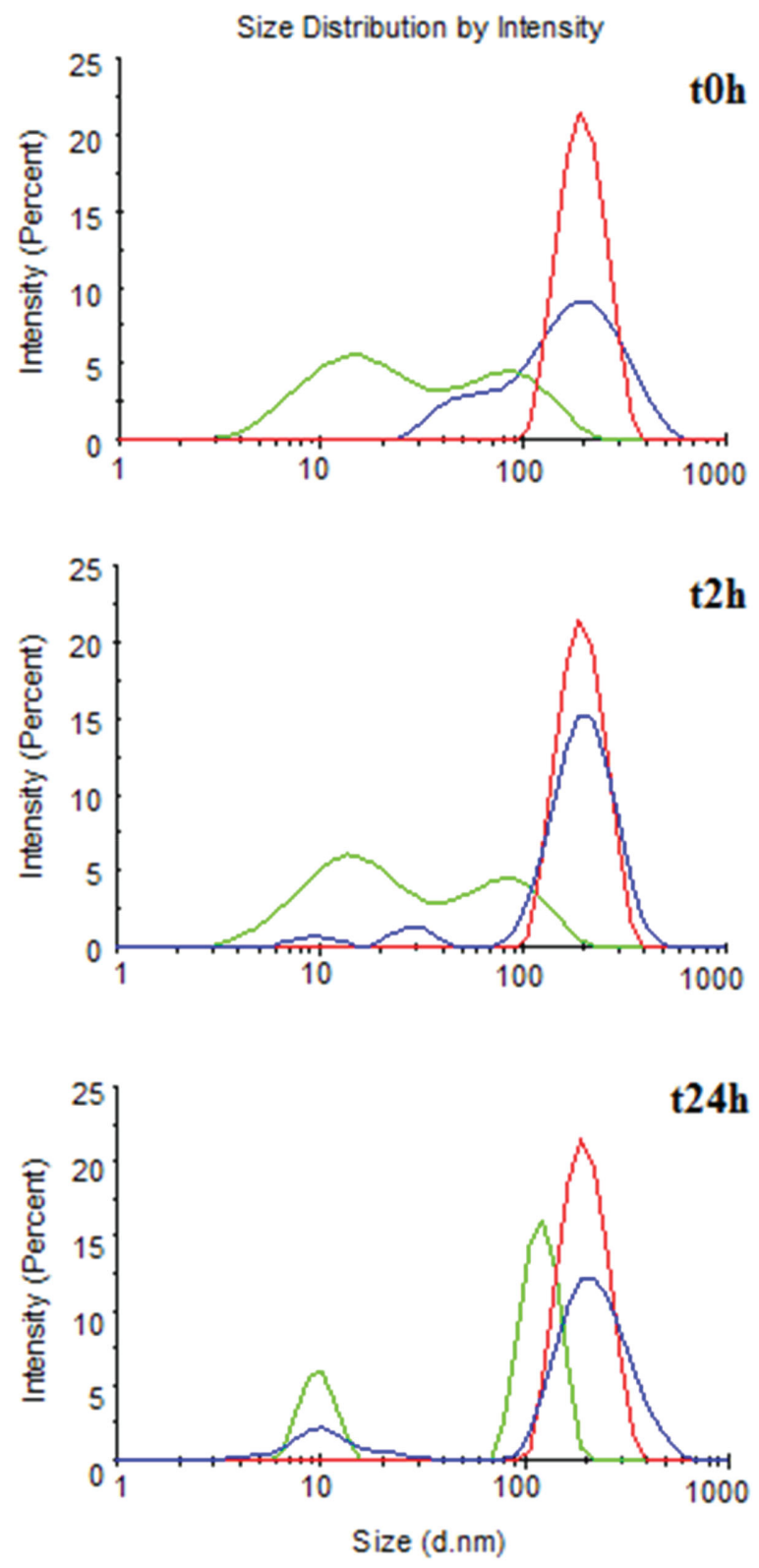

\section{- Nanogel - FBS - Nanogel + FBS}

Figure 1. Nanogel size stability in the presence of serum assessed by DLS over the time. 


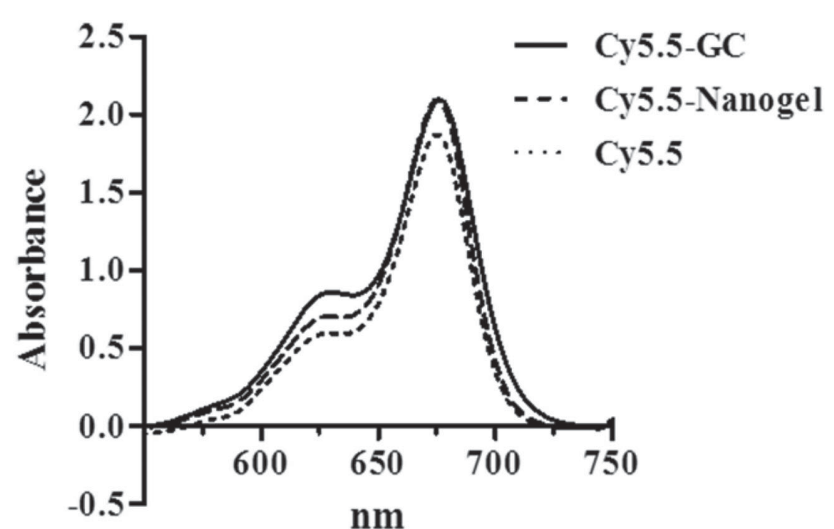

Figure 2. Absorbance spectral scans of Cy5.5, free and conjugated with GC or GC nanogel four times diluted as compared to injected samples.

of PEG to minimize nonspecific proteins adsorption and aggregation. ${ }^{[14]}$

\subsection{GC and Nanogel Labeling}

CyDyes are commonly used in a wide range of biological assays. Cy5.5 has been used historically for imaging, even though its excitation/emission wavelengths $(675 / 694 \mathrm{~nm})$ are very close to the wavelength range (400-650 nm, visible spectrum) which is affected by tissue autofluorescence. ${ }^{[15,16]}$ In order to comparatively study the in vivo biodistribution of the unmodified GC and its derived nanogel, Cy5.5 monoreactive ester was chosen to label both samples. The comparison is only possible if samples were similarly labeled. Therefore, a theoretical molar ratio of $4 \%$ and $6 \%$ of Cy5.5 reactive carboxylic groups in regard to the free amine groups, respectively for the GC polymer and its nanogel, was experimentally observed to provide a similar Cy5.5 labeling, as shown in Figure 2. Indeed, the resulting fluorescence signal obtained in the different samples is comparable.

The lack of unconjugated Cy5.5 in the labeled GC and nanogel was also assessed spectrophotometrically. Basal absorbance was recorded using the filtered fraction obtained following ultrafiltration, thus demonstrating that all of the conjugate is properly grafted on the polymer (data not shown).

\subsection{Unconjugated Cy5.5 Biodistribution}

The biodistribution/body clearance profile of free Cy5.5 was assessed $6 \mathrm{~h}$ post administration. Whole body imaging showed that free Cy5.5 was distributed to little extension, as compared to conjugated dye (Figure 3A), indicating faster body clearance. Negligible NIR fluorescence was detected in the blood, spleen, heart, kidneys, and brain (Figure 3B,C) using the free dye, a weak signal being found in the lungs, muscle, and liver. Remarkably, $6 \mathrm{~h}$ after injection an intense NIR fluorescence signal was observed in the skin, as also observed in animals treated with Cy5-5-GC or nanogel. Hue et al. ${ }^{[1]}$ found that Cy5.5 fluorescence in several organs was rapidly eliminated from $30 \mathrm{~min}$ to $24 \mathrm{~h}$ postinjection, fairly high fluorescence signal being reached in the liver, lung, kidneys, and stomach at the early time points.

\subsection{Cy5.5-GC and Cy5.5-Nanogel Biodistribution}

\subsubsection{Whole Body Biodistribution}

It is known that autofluorescence naturally occurs in animal tissues through the visible spectral range up to $700 \mathrm{~nm}$, which may mask the probe signal. ${ }^{[15]}$ Hence, this feature was taken into consideration and system image acquisition parameters were optimized using a noninjected mouse (Cont-). As could be observed in Figure 4, no interference of tissues autofluorescence was visualized under the used conditions. So, the NIR fluorescence signal detected by a CCD camera is exclusively associated with cy5.5.

In order to observe in vivo biodistribution of Cy5.5-GC and Cy5.5-Nanogel, a $5 \mathrm{mg} \mathrm{Kg}^{-1}$ dosage of the samples homogenously dispersed in $100 \mu \mathrm{L}$ of PBS was intravenously administered into the tail vein of BALB/c mice. A time-dependent distribution was observed using a noninvasive NIR fluorescence imaging technique in live animals, as shown in Figure 4. An intense NIR fluorescence signal was observed in the whole body 15 min only after injection (reflecting the rapid sample biodistribution), the signal remaining intense for at least $6 \mathrm{~h}$. The fluorescence signal fades drastically after $24 \mathrm{~h}$ postinjection. It is noteworthy that a strongest fluorescent signal was observed on the mice treated with Cy5.5-Nanogel and the distribution pattern clearly progresses from a widespread distribution at early time points to a more posterior concentrated distribution (kidneys and bladder) in a later stage, showing the predictable fate of the sample, its elimination by filtration. Likewise, in a similar study, the biodistribution profile of NPs of a $\mathrm{N}, \mathrm{N}$-diethylnicotinamide-based oligomer conjugated with GC showed high NIR fluorescent signal $1 \mathrm{~h}$ after injection, which was preserved for up to $1 \mathrm{~d}$, followed by a reduction. ${ }^{[18]}$

\subsubsection{Blood Clearance}

One of the major design considerations for nanoparticulate drug delivery systems is the circulation half-life, since the longer this is the more effectively the NPs may accumulate at the target site, either by passive or active mechanisms. ${ }^{[19]}$ Thus, in order to study the blood half-life, whole blood was collected from mice injected with the samples at

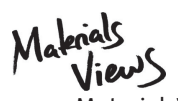

www.MaterialsViews.com 
(A)

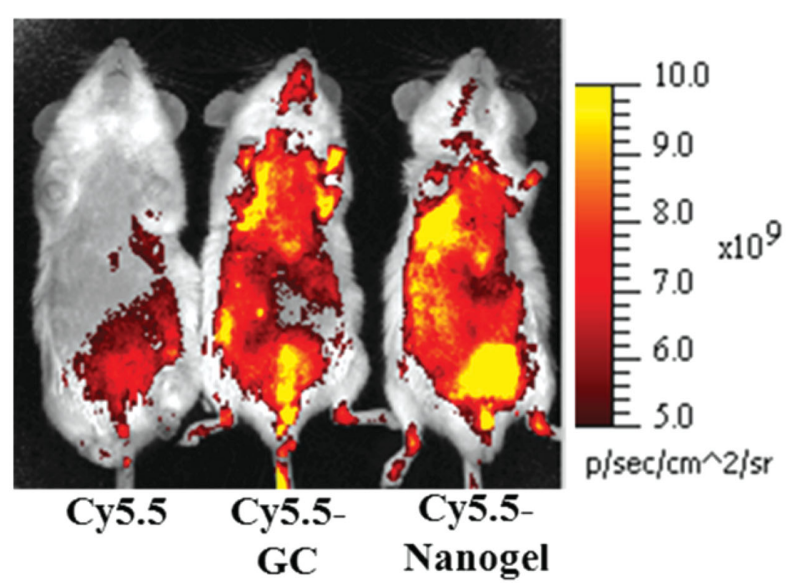

(B)

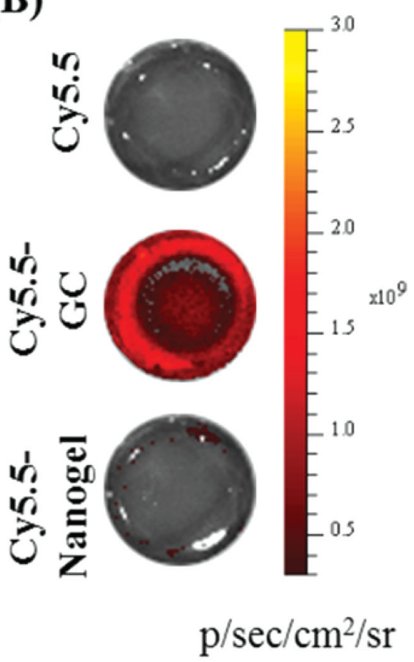

(C)

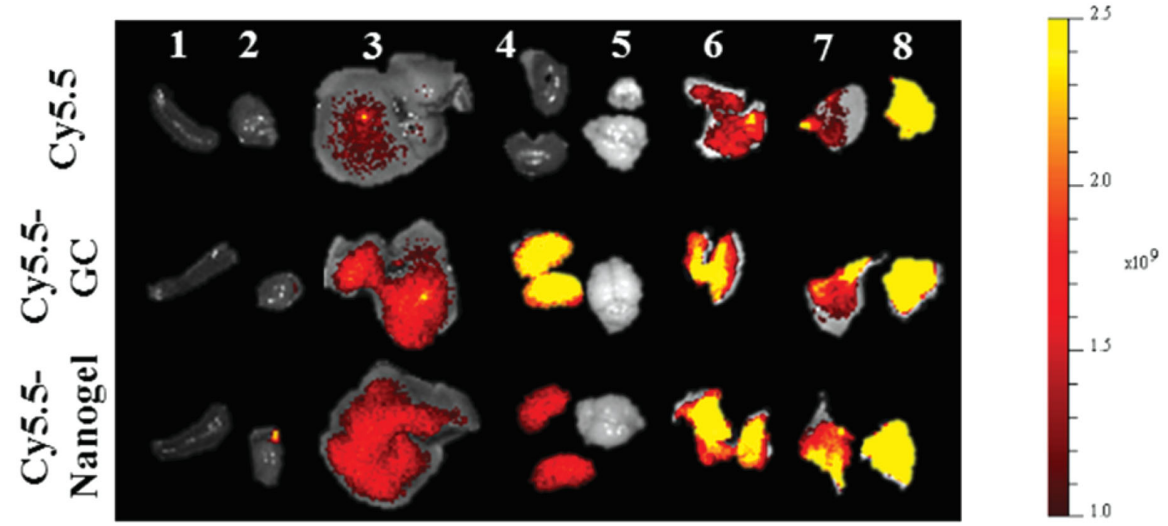

Figure 3. Biodistribution of unconjugated Cy5.5 as compared with Cy5.5 conjugated with GC or nanogel, in BALB/c mice $6 \mathrm{~h}$ postintravenous injection. A) NIR imaging of whole body, B) total blood, and C) ex vivo organs (1: spleen; 2: heart; 3: liver; 4: kidneys; 5: brain; 6: lungs; 7: muscle; and 8: skin).

different time points and scanned using the IVIS Lumina system (Figure 5). NIR fluorescence intensity of whole blood in each condition was semi-quantified using Living image software and expressed as average radiance. Liu et al. ${ }^{[10]}$ classify the fluorescence imaging as a semi-quantitative technique, as they proved that the quantitative data from whole organs are strongly affected by the scattering and the absorption properties of the organ. Therefore the fluorescence intensity detected may not necessarily be proportional to the number of molecules present, and thus the results are here discussed qualitatively.

The Cy5.5-Nanogel exhibited a fairly long blood circulation half-life, about $2 \mathrm{~h}$, which is compatible with the general aim of addressing NPs to a particular tissue in the body. Nevertheless, the NIR fluorescence intensity signal decays faster at the initial stage after administration, probably due to kidneys filtration and retention in the organs. Surprisingly, GC polymer presented a higher blood circulation half-life, being detected in significantly higher intensity than the nanogel $6 \mathrm{~h}$ postinjection.
In both cases, however, $24 \mathrm{~h}$ after administration only a residual amount is detectable. Apparently, the free polymer is thus more effective in evading the mononuclear phagocytic system. Foreign entities in bloodstream circulation are generally marked for uptake by mononuclear phagocytic system, through a process known as opsonization. Particles functionalized with PEG, or other hydrophilic polymers, have increased circulation half-life because they are shielded with water molecules, remaining invisible to opsonins and macrophages. ${ }^{[5,19]}$ Hence, lower circulation half-life of the GC nanogel comparatively to free polymer was unexpected, since the nanogel was decorated with PEG chains of 2000 and $3400 \mathrm{Da}$, whose ability for prolonging circulation time in blood is well reported. ${ }^{[20,21]}$ On the other hand, the size of the nanogel is much higher than that of the GC polymer. The GC used in this work has a molecular weight $\left(M_{\mathrm{w}}\right)$ of $100 \mathrm{kDa}$, expected to correspond to a hydrodynamic size of a few nanometers, while the nanogel has about $200 \mathrm{~nm}$. Hence a faster kidney filtration would be 

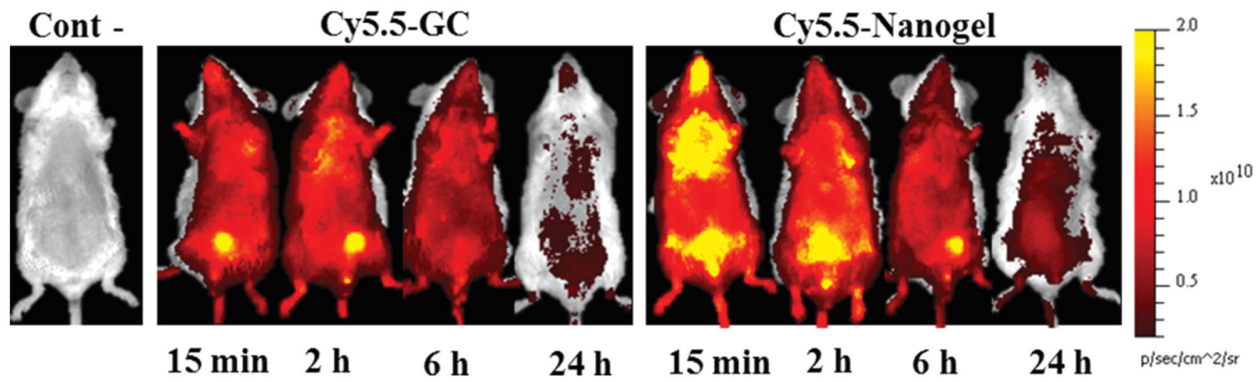

$15 \min 2 \mathrm{~h}$

$6 \mathrm{~h}$

$24 \mathrm{~h}$

Figure 4. Representative experiment of whole body NIR fluorescence images of Balb/C mice intravenously injected with Cy5.5-GC and Cy5.5-Nanogel (5 $\mathrm{mg} \mathrm{Kg}^{-1}$ ), observed over time.

expectable for the polymer. The larger size could on the other hand justify a more effective recognition of the nanogel by macrophages (although decorated with PEG) as the results consistently show the GC polymer has a longer blood circulation time. However, the accumulation of the nanogel in the liver, although occurring to larger extent than the free polymer, is only transient, as discussed below.

Also N-succinyl-chitosan was reported as a systemically long circulating polymer. The retention of succinylchitosan in blood was much higher than that in other tissues even at $72 \mathrm{~h}$ after injection. The half-life was calculated to be around $100 \mathrm{~h}$. Kato et al. argued that this longer retention was probably due to high $M_{\mathrm{w}}(>700 \mathrm{kDa})$, difficult biodegradation, and poor interaction with tissues due to its high negative charge, differently from the here analyzed nanogel..$^{[22]}$

Controversially, Richardson et al. reported rapid blood clearance for three tested samples of chitosan with different $M_{\mathrm{w}}(<5,5-10$, and $>10 \mathrm{kDa})$. In this study, $1 \mathrm{~h}$ after injection only $2.6 \%$ of the radiolabeled chitosan $\left({ }^{125} \mathrm{I}\right)$ with $>10 \mathrm{kDa}$ remains in the blood. ${ }^{[23]} \mathrm{Na}$ et al. ${ }^{[24]}$ verified that
GC NPs synthesized with increasing degrees of substitution of $5 \beta$-cholonic acid had higher blood circulation time compared to the GC linear polymer, whose fluorescence intensity decreased $1 \mathrm{~d}$ postinjection. Also Kim et al. ${ }^{[25]}$ found that Cy5.5 and Cy5.5 labeled GC were excreted from body within $1 \mathrm{~d}$, while hydrophobically modified GC takes $3 \mathrm{~d}$.

\subsubsection{Organs Biodistribution}

In order to evaluate the nanogel and linear GC organ distribution at defined time points, the organs were excised and imaged in the IVIS Lumina system. NIR fluorescence intensity of each organ was semi-quantified using Living image software and expressed as average radiance. Overall, GC nanogel shows higher tissue accumulation at earlier time points ( $t=15 \mathrm{~min}$ and $t=2 \mathrm{~h}$ ), mainly in the lungs and skin (highly vascularized organs) as compared to free polymer, as could be observed in Figure 6A,B. This observation is consistent with the shorter circulation half-life of the nanogel. Again, this is somewhat surprising, since the positive charges of the free polymer would be expected to
(A)

Cy5.5-GC Cy5.5-Nanogel

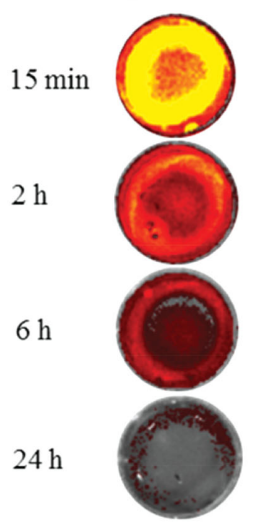

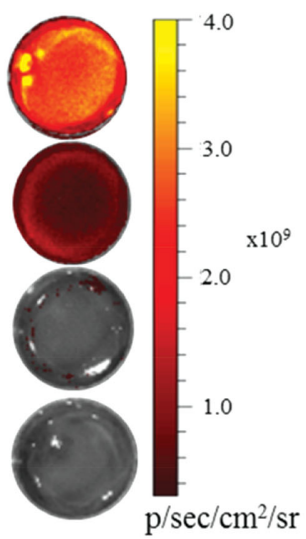

(B)

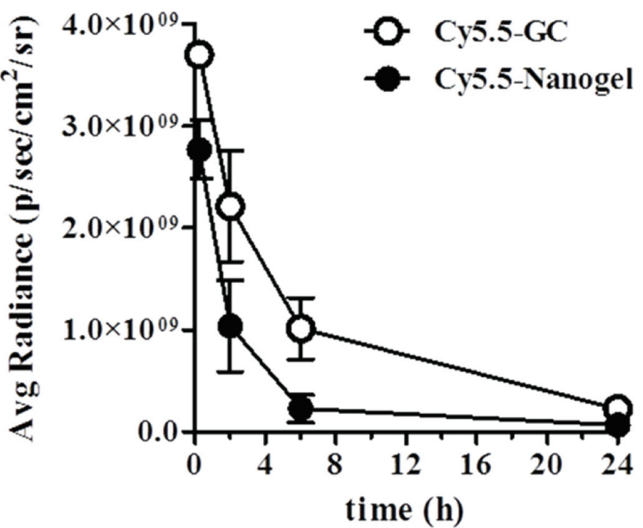

Figure 5. Blood circulation half-life of GC and nanogel. A) Representative NIR fluorescent images of whole blood collected over time after intravenous injection of Cy5.5-GC and Cy5.5-Nanogel samples in BALB/c mice. B) NIR fluorescence intensity signal quantification of Cy5.5 labeled samples.

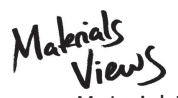

www.MaterialsViews.com 
(A) Cy5.5-GC

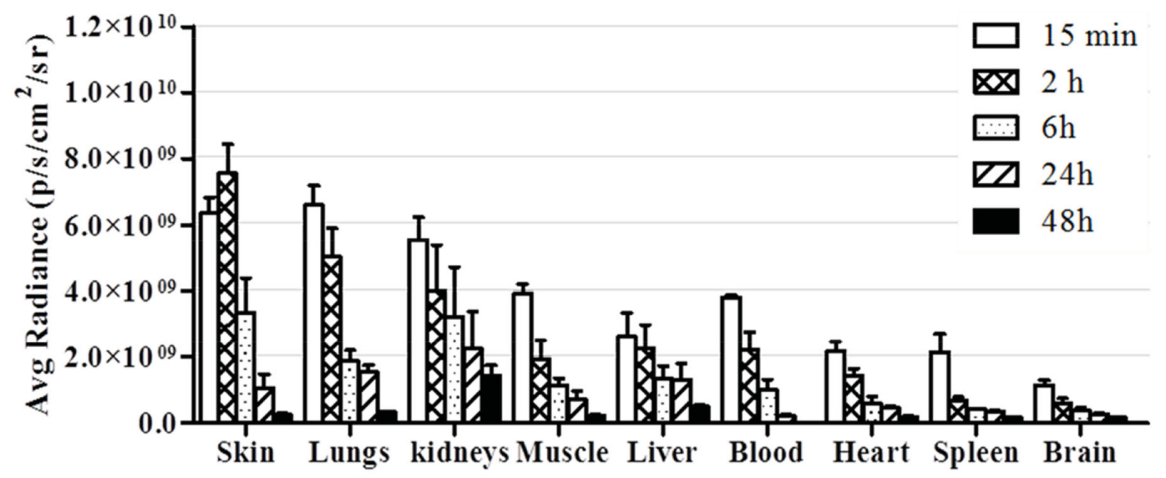

(B) Cy5.5-Nanogel

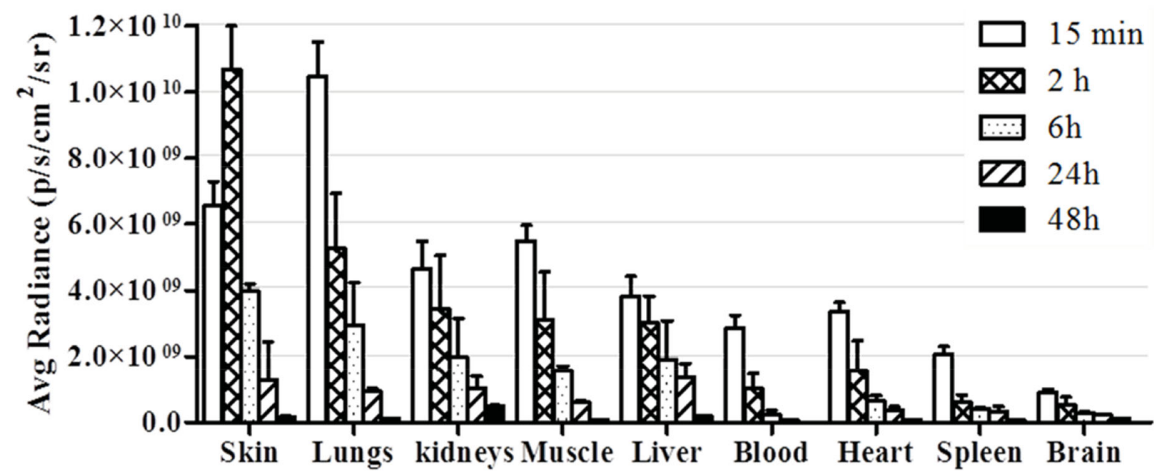

(C)

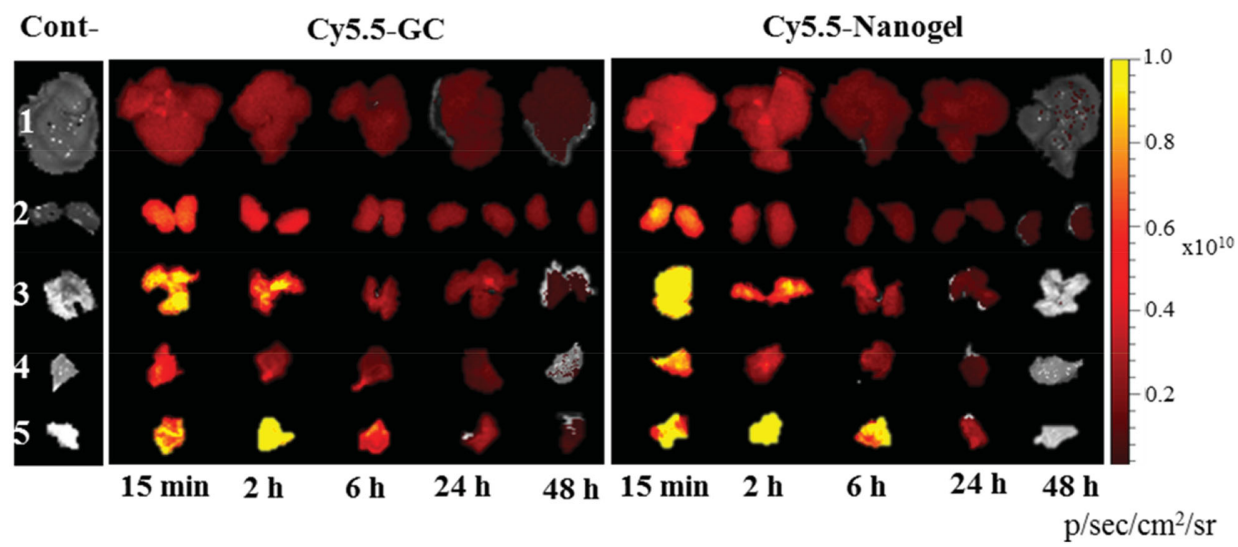

Figure 6. Ex vivo NIR fluorescence imaging of Cy5.5-GC and Nanogel organ biodistribution. A,B) Quantification of NIR fluorescence signal of Cy5.5-GC or Nanogel (respectively) organs accumulation at different time points, recorded as total photon counts per centimeter squared per steradian $\left(\mathrm{p} \mathrm{s}^{-1} \mathrm{~cm}^{-2} \mathrm{sr}^{-1}\right.$ ) per excised organ as a function of time. C) Representative ex vivo images of normal organs (1: liver; 2 : kidneys; 3: lungs; 4: muscle; and 5: skin) acquired over time after Cy5.5-GC or Nanogel intravenous injection. Organs of noninjected mice were used as negative control of NIR fluorescence.

interact with cells more readily than the nanogel, which is decorated with PEG. Maybe the folate receptor plays therefore a relevant role and it may be responsible for the quicker retention of the nanogel in the tissues. $24 \mathrm{~h}$ postinjection, when no GC or nanogel was observed in blood anymore, a similar organ distribution pattern was observed for both samples, exception made to higher accu- mulation of the free polymer in the lungs and kidneys, as observed in NIR images (Figure 6C). For later postinjection periods (48 h) higher NIR fluorescence signal was found in the organs of mice injected with linear GC as compared to nanogel, which barely was found, with exception to the kidneys. The nonspecific interaction of the free GC with cells seems to retard the excretion to some extent, 
as compared to the specific interactions mediated by the folate receptor. Localization of the nanogel and GC polymer in the kidneys is expected and common to other chitosan NPs because they play an important role in the clearance of biodegradable macromolecules circulating in the bloodstream. ${ }^{[25-27]}$ So, $48 \mathrm{~h}$ postinjection almost all of the injected nanogel was almost fully eliminated, which represents a great prospect concerning toxicity issues. This body clearance behavior suggests that the nanogel disassembles in vivo, being excreted in about 2-3 d. This is particularly important due to concerns over long-term exposure. ${ }^{[4]}$ The in vitro biocompatibility of this nanogel was reported in our previous manuscript. ${ }^{[28]}$ According to published works, the conjugated Cy5.5 should not bring about toxic-related issues. ${ }^{[29,30]}$

It should be noted that NIR signal fadeout corresponds indeed to the excretion of the conjugated dye, and not to the dye instability, or loss of signal over time. As a matter of fact, similar studies such as biodistribution of Cy5.5 labeled chitosan coated iron oxide NPs showed high signal intensity for at least $72 \mathrm{~h}$ in the kidneys, spleen, liver, and bone marrow. ${ }^{[5]}$ Based on this observation, an interesting approach for kidneys targeted drug delivery was developed by Gao et al. ${ }^{[31]}$ They reported for the first time the use of chitosan/siRNA NPs for extended siRNA accumulation in the kidneys, which may have potential for treatment of renal diseases using RNAi therapeutics.

Curiously, in spite of the liver, spleen, and lung being important components of the mononuclear phagocytic system and consequently involved in macromolecules clearance, in the current study nanogel and even free GC were poorly taken up by the liver and spleen, unlike described by other authors. ${ }^{[5,32]}$

Nevertheless also He et al. ${ }^{[33]}$ verified that rhodamine B labeled chitosan hydrochloride distribution in spleen decreased with the increase of the particle size from 150 to $300 \mathrm{~nm}$, which was probably due to the splenic physical filtration effect that excluded NPs with particle size ranging in $200-500 \mathrm{~nm}$. Indeed, the size distribution of the GC nanogel studied in the present work lies within this range, so it could be predicted that this nanogel with an average size of $220 \mathrm{~nm}$ would show minor accumulation in the spleen.

Lungs, together with skin, is one of the organs where higher nanogel accumulation was found $24 \mathrm{~h}$ postadministration, as also the free polymer-although to less extent in this case. He et al. ${ }^{[33]}$ assigned the higher concentration of rhodamine B labeled chitosan hydrochloride in the lungs to the high positive charge, which would lead to the NPs forming aggregates with blood cells by electrostatic interaction, consequently being entrapped in the lungs. Also Knudsen et al. ${ }^{[34]}$ found that cationic liposomes after intravenous administration were preferentially distributed to the lungs. Sykes et al. $^{[35]}$ also showed that skin is an important site of NPs accumulation following systemic administration. Their results suggest that dermal accumulation should be exploited to trigger the release of ultraviolet and visible light-sensitive therapeutics which are currently impractical in vivo. Nevertheless, further tests should be performed in order to clarify whether the GC and nanogel skin accumulation $24 \mathrm{~h}$ postinjection are independent of the dye, because as shown ahead in Figure 3 fairly high NIR fluorescence intensity was observed in this tissue $6 \mathrm{~h}$ after free dye administration.

\section{Conclusion}

The rapid biodistribution of GC nanogel and linear GC labeled with Cy5.5 was readily observed through in vivo NIR imaging system. Whole body images acquisition provides an overview on the extension of sample biodistribution, however it is the whole blood and organs analysis that renders more accurate information on nanogel blood clearance, organs accumulation, and consequently also on the body clearance. In summary, it could be concluded that the nanogel has a blood clearance rate superior to that of the free polymer. Even so, the nanogel exhibits a satisfactory blood circulation half-life of about $2 \mathrm{~h}$ and its body clearance occurs $\approx 48 \mathrm{~h}$ after administration.

Acknowledgements: The authors thank the FCT Strategic Project of UID/BIO/04469/2013 unit, the project RECI/BBB-EBI/0179/2012 (FCOMP-01-0124-FEDER-027462), and the Project "BioHealthBiotechnology and Bioengineering approaches to improve health quality," Ref. NORTE-07-0124-FEDER-000027, co-funded by the Programa Operacional Regional do Norte (ON.2-O Novo Norte), QREN, FEDER. The authors also thank António Temudo, Dolores Bonaparte, and Sílvia Santos Pedrosa for the support on in vivo assays. Paula Pereira acknowledges FCT for the PhD grant SFRH/ $\mathrm{BD} / 64977 / 2009$.

Received: July 14, 2015; Revised: November 11, 2015; Published online: December 10, 2015; DOI: 10.1002/mabi.201500267

Keywords: biodistribution; blood clearance; Cy5.5; glycol chitosan; nanogel; NIR imaging

[1] R. A. Petros, J. M. DeSimone, Nat. Rev. Drug Discovery 2010 , 9, 615.

[2] D.-E. Lee, H. Koo, I.-C. Sun, J. H. Ryu, K. Kim, I. C. Kwon, Chem. Soc. Rev. 2012, 41, 2656.

[3] T. Nam, S. Park, S.-Y. Lee, K. Park, K. Choi, I. C. Song, M. H. Han, J. J. Leary, S. A. Yuk, I. C. Kwon, K. Kim, S. Y. Jeong, Bioconjugate Chem. 2010, 21, 578.

[4] M. A. Phillips, M. L. Gran, N. A. Peppas, Nano Today 2010, 5, 143.

[5] M. J. Lee, O. Veiseh, N. Bhattarai, C. Sun, S. J. Hansen, S. Ditzler, S. Knoblaugh, D. Lee, R. Ellenbogen, M. Zhang, J. M. Olson, PloS One 2010, 5, e9536.

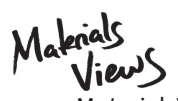

www.MaterialsViews.com 
[6] P. Pereira, D. Morgado, A. Crepet, L. David, F. M. Gama, Macromol. Biosci. 2013, 13, 1369.

[7] D. Torrecilla, M. V. Lozano, E. Lallana, J. I. Neissa, R. Novoa-Carballal, A. Vidal, E. Fernandez-Megia, D. Torres, R. Riguera, M. J. Alonso, F. Dominguez, Eur. J. Pharm. Biopharm. 2013, 83, 330.

[8] H. L. Osterman, A. Schutz-Geschwender.

[9] C.-H. Lee, S.-H. Cheng, Y.-J. Wang, Y.-C. Chen, N.-T. Chen, J. Souris, C.-T. Chen, C.-Y. Mou, C.-S. Yang, L.-W. Lo, Adv. Funct. Mater. 2009, 19, 215.

[10] Y. Liu, Y. C. Tseng, L. Huang, Pharm. Res. 2012, 29, 3273.

[11] H. S. Choi, B. I. Ipe, P. Misra, J. H. Lee, M. G. Bawendi, J. V. Frangioni, Nano Lett. 2009, 9, 2354.

[12] O. Lin, J. Chen, Z. Zhang, G. Zheng, Nanomedicine 2014, 9, 105.

[13] L. Nuhn, S. Gietzen, K. Mohr, K. Fischer, K. Toh, K. Miyata, Y. Matsumoto, K. Kataoka, M. Schmidt, R. Zentel, Biomacromolecules 2014, 15, 1526.

[14] Y. Li, R. Liu, Y. Shi, Z. Zhang, X. Zhang, Theranostics 2015, $5,583$.

[15] K. E. Adams, S. Ke, S. Kwon, F. Liang, Z. Fan, Y. Lu, K. Hirschi, M. E. Mawad, M. A. Barry, E. M. Sevick-Muraca, J Biomed Opt 2007, 12, 024017.

[16] H. Y. Hwang, I. S. Kim, I. C. Kwon, Y. H. Kim, J. Controlled Release 2008, 128, 23.

[17] J. J. Hue, H. J. Lee, S. Jon, S. Y. Nam, Y. W. Yun, J. S. Kim, B. J. Lee, J. Vet. Sci. 2013, 14, 473.

[18] G. Saravanakumar, K. H. Min, D. S. Min, A. Y. Kim, C. M. Lee, Y. W. Cho, S. C. Lee, K. Kim, S. Y. Jeong, K. Park, J. H. Park, I. C. Kwon, J. Controlled Release 2009, 140, 210.

[19] R. H. Fang, C. M. Hu, L. Zhang, Expert Opin. Biological Therapy 2012, 12, 385.

[20] Y. Sheng, C. Liu, Y. Yuan, X. Tao, F. Yang, X. Shan, H. Zhou, F. Xu, Biomaterials 2009, 30, 2340.

[21] Z. Hou, C. Zhan, O. Jiang, O. Hu, L. Li, D. Chang, X. Yang, Y. Wang, Y. Li, S. Ye, L. Xie, Y. Yi, O. Zhang, Nanoscale Res. Lett. 2011, 6, 563
[22] Y. Kato, H. Onishi, Y. Machida, Biomaterials 2000, 21, 1579.

[23] S. C. Richardson, H. V. Kolbe, R. Duncan, Int. J. Pharm. 1999, 178, 231.

[24] J. H. Na, S. Y. Lee, S. Lee, H. Koo, K. H. Min, S. Y. Jeong, S. H. Yuk, K. Kim, I. C. Kwon, J. Controlled Release 2012, 163, 2.

[25] J.-H. Kim, Y.-S. Kim, K. Park, S. Lee, H. Y. Nam, K. H. Min, H. G. Jo, J. H. Park, K. Choi, S. Y. Jeong, R.-W. Park, I.-S. Kim, K. Kim, I. C. Kwon, J. Controlled Release 2008, 127, 41.

[26] V. H. Pereira, A. J. Salgado, J. M. Oliveira, S. R. Cerqueira, A. M. Frias, J. S. Fraga, S. Roque, A. M. Falcão, F. Marques, N. M. Neves, J. F. Mano, R. L. Reis, N. Sousa, J. Bioactive Compatible Polymers 2011, 26, 619.

[27] S. J. Lee, M. S. Huh, S. Y. Lee, S. Min, S. Lee, H. Koo, J. U. Chu, K. E. Lee, H. Jeon, Y. Choi, K. Choi, Y. Byun, S. Y. Jeong, K. Park, K. Kim, I. C. Kwon, Angew. Chem. Int. Ed. Engl. 2012, 51, 7203.

[28] P. Pereira, S. S. Pedrosa, A. Correia, C. F. Lima, M. P. Olmedo, A. Gonzalez-Fernandez, M. Vilanova, F. M. Gama, Toxicol. In Vitro 2015, 29, 638.

[29] K. Kim, M. Lee, H. Park, J. H. Kim, S. Kim, H. Chung, K. Choi, I. S. Kim, B. L. Seong, I. C. Kwon, J. Am. Chem. Soc. 2006, 128, 3490.

[30] R. Alford, H. M. Simpson, J. Duberman, G. C. Hill, M. Ogawa, C. Regino, H. Kobayashi, P. L. Choyke, Mol. Imaging 2009, $8,341$.

[31] S. Gao, F. Dagnaes-Hansen, E. J. Nielsen, J. Wengel, F. Besenbacher, K. A. Howard, J. Kjems, Mol. Therapy 2009, $17,1225$.

[32] S. P. Egusquiaguirre, N. Beziere, J. L. Pedraz, R. M. Hernández, V. Ntziachristos, M. Igartua, Contrast Media Mol. Imaging 2015, DOI: 10.1002/cmmi.1644.

[33] C. He, Y. Hu, L. Yin, C. Tang, C. Yin, Biomaterials 2010, 31, 3657.

[34] K. Knudsen, H. Northeved, T. Gjetting, A. Permin, T. Andresen, K. Wegener, H. Lam, J. Lykkesfeldt, J. Nanopart. Res. 2014, 16, 1.

[35] E. A. Sykes, O. Dai, K. M. Tsoi, D. M. Hwang, W. C. W. Chan, Nat. Commun. 2014, 5, 3796. 